\title{
Dynamic Stability and Control Design of Modern Electric Energy Systems with Large Penetration of Distributed Generators
}

\author{
M. Honarvar Nazari, IEEE Member, M. Ilić, IEEE Fellow, and J. Peças Lopes, IEEE Senior Member
}

\begin{abstract}
This paper investigates dynamic stability and control design of modern electric energy systems as many MWscale distributed generators (DG) of various types are deployed in the distribution side of electric systems. The paper is mainly concerned about small-signal frequency stability of distribution systems with decentralized or centralized control systems. The Gerschgorin Circle Theory and Hicks Stability Theory are used for verifying sufficient conditions for small-signal stability. The paper also discusses about designing advanced enhancing robustness methods to ensure both safety and stability of modern distribution systems. Finally, the paper closes with a discussion of policies needed to support large penetration of DG units in distribution systems to ensure safety and robustness of the system.
\end{abstract}

Index Terms - AC Optimal Power Flow (OPF), Advanced Control Design, Distributed Generation, Dynamic stability, Modern Distribution Systems, Gerschgorin Circle Theory, Hicks Stability Theory, Small-Signal Frequency Instability, Robustness.

\section{INTRODUCTION}

$\mathrm{N}$ EW pressures for more sustainable, cleaner, more efficient and simply smarter use of energy have led to large deployment of smaller-scale power plants closer to the end users. These units are broadly referred to as distributed generation (DG) and they offer many advantages to electric power systems. For instance, Combined Heat \& Power (CHP) DG units offer the advantage of increased efficiency through waste heat recovery, and high reliability and security (Zerrifi et al [1]). Low-head hydro, wind machines and solar arrays provide electricity from renewable sources. All DG systems also hold the potential to reduce transmission and distribution losses.

Nevertheless, for large integration of MW-scale DG systems into legacy distribution systems, it is essential to: 1) evaluate current operating and planning practices in order to best integrate and utilize new distributed energy resources; 2 ) identify potential technical challenges brought about by large integration of DG systems in electric power systems; and finally, 3) introduce technically innovative ways and theoretically sufficient conditions for facilitating the best integration of DGs without creating reliability and safety problems. This paper tries to address all three of these issues, demonstrating results on a representative electric power distribution system. It is worth mentioning that among all the technical problems may occur by extensive integration of DGs, small-signal frequency instability of distribution networks is the main concern of this paper.

Here first the plausible structure of modern distribution systems is demonstrated when DG units provide large portion of electricity and therefore the role of centralized power plants diminishes [2]. In section II it is illustrated how such a structure can potentially improve efficiency of electric power systems.

In section III it is shown that large penetration of DG units sending power into the electric distribution systems may cause small-signal frequency instability problems not being observed in the old systems with one power plant. This new phenomenon has been recently observed by several authors including Cardell et al [3] and [4], Lopes et al [5], Guttromoson [6] and Donnelly et al [7]. However, the explanation of the cause of these problems and the identification of solutions to deal with them still needs further investigation.

In Section III the frequency stability of a representative electric power distribution system is explored when DG units supply more than $10 \%$ of the electricity. Moreover, in section III the conditions in which frequency instabilities occur are investigated. Then, the nature of instabilities is identified by using Gerschgorin Circle Theory. The Gerschgorin Circle Theory is also applied to determine sufficient conditions for frequency stability of distribution systems with decentralized control systems.

Based on these findings, it is concluded that in order to ensure frequency stability of distribution systems with decentralized controls, each DG needs to be locally stable and also the strength of its electrical interaction with the rest of the system (other DGs in the system) should be weaker than the damping magnitude of the slowest eigenmode of the DG. Moreover, given a system of interest and candidate DGs, it is possible to derive the minimum electrical distance bound beyond which the system remains stable. These criteria could be used as guideline methods for approving placement of new DGs, which ensures robust of the system. 
In Section IV dynamic stability of modern electric energy systems equipped with centralized control systems is investigated. Sufficient conditions for frequency stability of these systems are also extracted by using Hicks Stability Theory. The findings demonstrate that it is essential for systems with centralized control systems to be fully controllable and observable.

Finally, the technical results induce questions regarding policy making and institutional arrangements for integrating in technically feasible ways of sitting and operating many larger DGs in the legacy distribution networks. Hence, Section V concisely identifies and discusses policy issues, which must be dealt with in order to make this feasible.

\section{Modern Electric ENERGy Systems}

Traditionally, electric power systems have had vertical structures in which electricity is produced by large central power plants and transferred to the end users through transmission and distribution networks. Nevertheless, this structure presents some major drawbacks for utilities, consumers and environment. For instance, traditional power systems have low efficiency in producing and transferring electric power ${ }^{1}$, they have negative environmental impacts from large coal power plants and transmission lines, and also costs of enlarging transmission and distribution networks is very high. Consequently, such electric power systems have been transforming into modern electric energy systems with many new distributed generators on the distribution side of the network. Figure 1 shows a schematics of such a modern distribution system with new candidate DGs.

When larger DGs are responsible for supplying significant portion of system needs, it is essential that these units are equipped with proper frequency control systems. In fact, in most of the European countries such as Germany and Portugal it is highly recommended for large DGs to be equipped with governor-control (G-C) systems and participate in primary frequency control. In addition, in the next section it is shown that droop constants of DG units equipped with governorcontrol systems are significantly lower than that of DGs without G-C systems.

\section{A. Droop Constant}

In general "... The droop constant represents the sensitivity of the steady-state frequency to the real-power output of a generator when the frequency setting is kept constant ..." [13]. Equation (1) shows how the droop constant $(\Sigma)$ of a generator is calculated based on the sensitivity of frequency $\left(\omega_{G}\right)$ to the real power $\left(P_{G}\right)[13]$.

$$
\Sigma=-\frac{\partial \omega_{G}[k]}{\partial P_{G}[k]_{\omega_{G}^{r e f}[k]=0}}
$$

When the droop constant of DGs shown in Figure 1 is

\footnotetext{
${ }^{1}$ Efficiency of centralized power plant is $30 \%$ whereas efficiency of Combined Heat and Power (CHP), which is one form of DG, is more than $95 \%$.
}

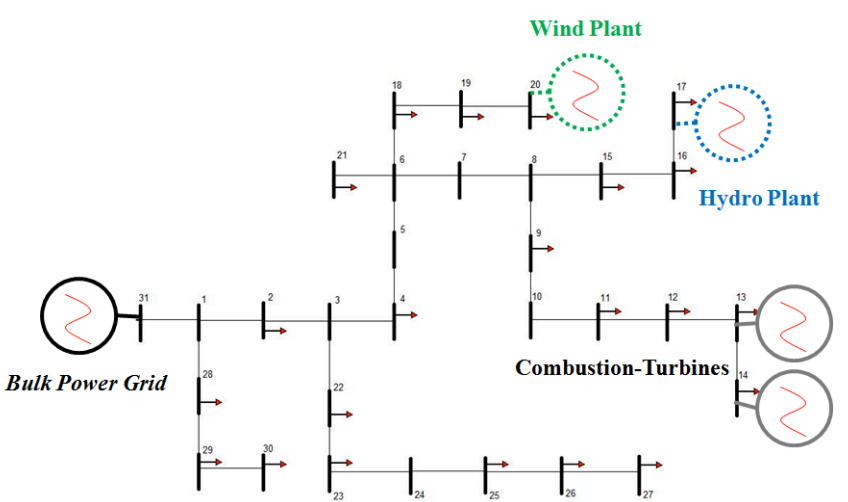

Fig. 1. Schematic of a typical future distributed energy system. For demonstration, shown in black is a point of connection between the transmission and distribution system modeled as an ideal power source. Represented in grey are two Combustion-Turbines (C-T) connected to nodes 13 and 14.

calculated with and without closed loop frequency control system, it is revealed that DGs without closed loop controls have significantly higher droop constants. For instance, based on the equation (1) the droop constant of C-Ts with G-C is calculated as 0.075 p.u and that of C-Ts without G-C is measured as 0.5 p.u. This implies that in order to minimize the sensitivity of the frequency to the real power, at least larger DGs need to be equipped with closed loop G-C systems. The parameters of C-Ts are also extracted from [4] and represented in Table B.1.

Nevertheless, it is explained in the next section of this paper that while G-Cs of DGs are adjusted for optimal steady state utilization and minimal droop constants, the system could become very sensitive (non-robust) even to very small fluctuations around operating points. In particular, it is shown that strong interaction between DG units or low damping magnitude of DGs could lead to small-signal frequency instabilities in the modern distribution systems. It is critical to observe that such analysis do not carry at traditional distribution companies, since in the old systems with one power plant this was not a possible scenario at all.

\section{DeCentralized Control Design For Modern DISTRIBUTED ENERGY SYSTEMS}

In this section frequency stability of future distribution systems with DGs equipped with decentralized control systems is assessed. Here the sufficient conditions for stability of such evolving distribution systems are identified using Gerschgorin Circle Theory. Earlier work has indicated that potential small-signal frequency instabilities may occur in distribution networks with large penetration of MW-scale DG units [3][7] and [14].

This paper stresses that it is possible to identify fundamental causes of potential frequency instabilities in terms of the strength of electrical interaction between DGs and the damping magnitude of the state variables of DG units. To this end, the paper first creates the dynamic model of the whole interconnected system by coupling the dynamic model 
of DGs to each other via the distribution network. As a result, a linearized state-space model of all segments of the network is developed. The first dynamic model being presented is the state-space model of local DG units. This model is extracted by linearizing the state-space model of DGs around their equilibrium point. In general, the dynamic model of DG units has the following structure [4]:

$\frac{d \bar{x}_{L C}}{d t}=A_{L C} \bar{x}_{L C}+C_{M} \bar{P}_{G}$

where $\bar{x}_{L C}$ is the local state vector of the DG, $\boldsymbol{A}_{L C}$ is the local system matrix whose elements consist of the linear coefficients of DG's parameters, $\bar{P}_{G}$ is the vector of coupling variables, and $C_{\mathrm{M}}$ is the matrix of coupling variables whose elements are zero except for that corresponding to $\omega_{G}$. The non-zero element of $C_{\mathrm{M}}$ is also equal to $-\frac{1}{M}$, where $M$ is the inertia of the DG. This model has been developed for the first time in [4].

In the next step, the dynamic model of DGs is connected to each other via the interconnected system variable. The interconnected variable is chosen as the active power outputs of generators, $P_{G}$, and the state-space equation for which is as following [16];

$\frac{d \bar{P}_{G}}{d t}=K_{P} \bar{\omega}_{G}+D_{P} \frac{d \bar{P}_{L}}{d t}$

where $\frac{d \bar{P}_{L}}{d t}$ represents load disturbance and $K_{P}$ and $D_{P}$ are coupling and disturbance matrices respectively, defined as following:

$$
\begin{gathered}
K_{P}=J_{G G}-J_{G L} J_{L L}^{-1} J_{L G} \\
D_{P}=-J_{G L} J_{L L}^{-1}
\end{gathered}
$$

The $J_{i j}$ is also defined as $\partial P_{i} / \partial \delta_{j}$ with $\mathrm{i}$ and $\mathrm{j}=\mathrm{G}$ (generator buses) and L (load buses). The model in (3) was first introduced in [16] and used for the first time in [4].

By adding (3) to (2) and expanding the model to the general form, the full system model with $n$ DGs in the system takes the following form [4]:

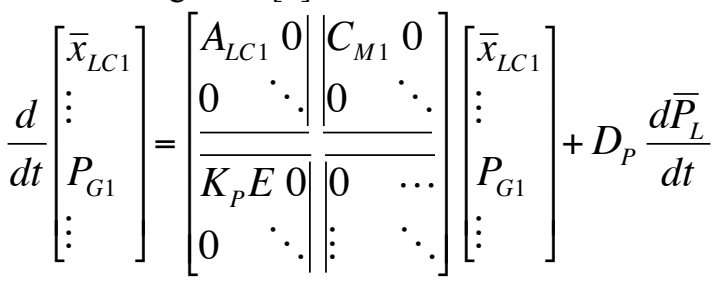

This model was also introduced for the first time in [4]. However, [4] did not represent the decentralized characteristics of modern distribution systems. As a result, in order to better analyze dynamic stability of these systems, here the model of (4) is modified by changing the order of state variables and introducing new sub-systems whose state variables are the local state variables of DGs and the active power out of the corresponding DG. Therefore, the new system model takes the following structure: $\frac{d}{d t}\left[\begin{array}{l}\bar{X}_{1} \\ \vdots\end{array}\right]=\left[\begin{array}{ccc}A_{11} & A_{12} & \cdots \\ A_{21} & A_{22} & \cdots \\ \vdots & \vdots & \ddots\end{array}\right]\left[\begin{array}{l}\bar{X}_{1} \\ \vdots\end{array}\right]+D_{P} \frac{d \bar{P}_{L}}{d t}$

where

$$
\bar{X}_{i}=\left[\begin{array}{l}
\bar{x}_{L C_{i}} \\
P_{G_{i}}
\end{array}\right] \quad A_{i i}=\left[\begin{array}{cc}
A_{L C_{i}} & C_{M_{i}} \\
K_{P_{i i}} & 0
\end{array}\right] \quad A_{i j}=\left[\begin{array}{ll}
0 & 0 \\
K_{P_{i j}} & 0
\end{array}\right]
$$

As noted earlier, the particular interest is to identify sufficient conditions for dynamic stability of future distribution systems. Thus, using Gerschgorin Circle Theorem, which has been fully explained in [17], the following inequality holds for all the sub-systems of (5):

$$
\left(\left\|\left(A_{i i}-z I_{i}\right)^{-1}\right\|\right)^{-1} \leq \sum_{\substack{j=1 \\ j \neq i}}^{n}\left\|A_{i j}\right\| \quad \forall i \in[1, n]
$$

The left hand side of (6) represents the set of complex numbers that all the eigenvalues of the full system matrix lie in the union of these sets [17]. Also, the right hand side of (6) represents the sum of coupling variables between the $i$ th DG and other DGs in the system. Hence, (6) can be re-written as following;

$$
\min \left\{\left|z-\lambda_{i 1}\right| \cdots\left|z-\lambda_{i n}\right|\right\} \leq \sum_{\substack{j=1 \\ j \neq i}}^{n}\left|K_{P_{i j}}\right| \forall i \in[1, n]
$$

Equation (7) illustrates that eigenvalues of the full system lie in the circles with centers equal to the eigenvalues of subsystems and with the radius equal to the sum of the electrical interaction between DGs. Figure 2 demonstrates the schematic of the circles in which eigenvalues of the full system lie. The blue crosses represent the eigenvalues of subsystems $\left(A_{i i}\right)$.

One can simply imply from Figure 2 that the stability of the system is satisfied when all the above-mentioned circles lie in the left hand side of the complex plane. That is;

1) Eigenvalues of all sub-systems lie in the left hand side of the complex plane (sub-systems are asymptotically stable)

2) The real part of the slowest eigenvalue of each sub-system

(the closest eigenvalue to the imaginary axis) is larger than the sum of the electrical distance between the DG and other DGs.

The physical interpretation of this theory is that when local DGs are asymptotically stable and the strength of electrical interaction (sum of electrical distances) between DGs is less than the damping magnitude of the slowest eigenmode of DGs, then the whole system always remain asymptotically stable.

This also implies that the main cause of frequency instability in distribution networks with high penetration of DGs is: 1) low damping magnitude of the state variables of local DGs 2) strong coupling between DGs. In general, low damping magnitude results from low inertia of DGs or poor tuning of their governor controls. Furthermore, strong coupling between DGs is caused by short electrical distance between DGs. In other words, low resistance between DGs 


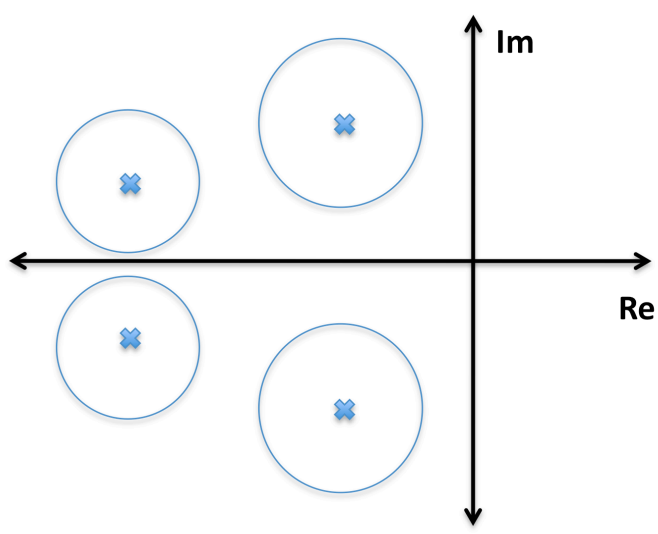

Fig. 2. Illustration of the dynamic response of the system with two C-Ts when DGs are located at the optimum locations and their inertia has been increased tenfold.

results in the low damping magnitude of local modes and therefore it leads to local mode of oscillation in the system.

To explore these facts, here we investigate small-signal stability of the representative distribution network shown in Figure 1 when two C-T generators equipped with decentralized G-C systems. Dynamic model of the C-T generators is also presented in Appendix B.

The exhaustive small-signal study on 900 possible combinations of locating two C-Ts in the 30-node distribution system shows that 192 combinations of locating DGs are small signal unstable. That is, in 192 cases the system matrix has positive eigenvalues. It is worth mentioning that the optimum location with respect to loss minimization is also among unstable cases. Figure 3 shows how the frequency deviation of C-Ts placed at optimum locations becomes unstable. Moreover, the results show that none of the unstable cases are satisfying the sufficient conditions for stability. Analytical results of these cases are presented in Appendix A.

Furthermore, the results demonstrate that in general designing proper decentralized control systems for future distribution systems can ensure frequency stability of these systems. However, a proper control design is often very system-dependent since the stability criteria depends on many parameters such as the location of DG units in the system, DGs' technology, their inertia, the system topology, and the equilibrium point of the system. Moreover, DGs need to be locally controllable so that designing proper control systems satisfies the sufficient conditions for stability.

In order to improve dynamic stability of modern distribution systems, this paper recommends that at least MWscale DG units need to be equipped with advanced decentralized control systems which can place poles of DGs in the locations where the sufficient conditions for frequency stability of the whole system are satisfied. Equation (8) illustrates the mathematical representation of such decentralized control systems.

$$
\frac{d \bar{X}_{i}}{d t}=A_{i i} \bar{X}_{i}+B_{i} \bar{U}_{i}+\sum_{\substack{j=1 \\ j \notin i}}^{n} A_{i j} \bar{X}_{j} \forall i \in[1, n]
$$

where $\bar{U}_{i}$ is the decentralized control signal of the $i$ th subsystem and $B_{i}$ is the control matrix. Furthermore, the control signal is a state feedback controller whose gain $\left(K_{i}\right)$ is calculated based on the desire poles of the sub-system.

$$
\bar{U}_{i}=-K_{i} \bar{X}_{i}
$$

In general, decentralized control systems give much flexibility to distributed generators and have many advantages for modern electric systems such as:

1. Simpler control systems;

2. No communication issue;

3. No sensing issue; and,

4. Cheaper control design

Implementing decentralized control systems also reduces complexity of controls. The paper also shows in the next section that without advanced decentralized control systems, future distribution systems need to be equipped with advanced centralized communication and observation systems to warrant frequency stability. Decentralized control systems nevertheless have some inherent drawbacks for future energy systems such as:

1. Potential sub-optimality (more MW loss); and,

2. Needs to ensure that decentralized controls do what they need to do

AMI (Automatic Meter Infrastructure) can resolve the latter problem; however, due to delays in communication these technologies are not effective for primary control purpose. Furthermore, the former drawback of decentralized control systems is unavoidable due to the inherent sub-optimal

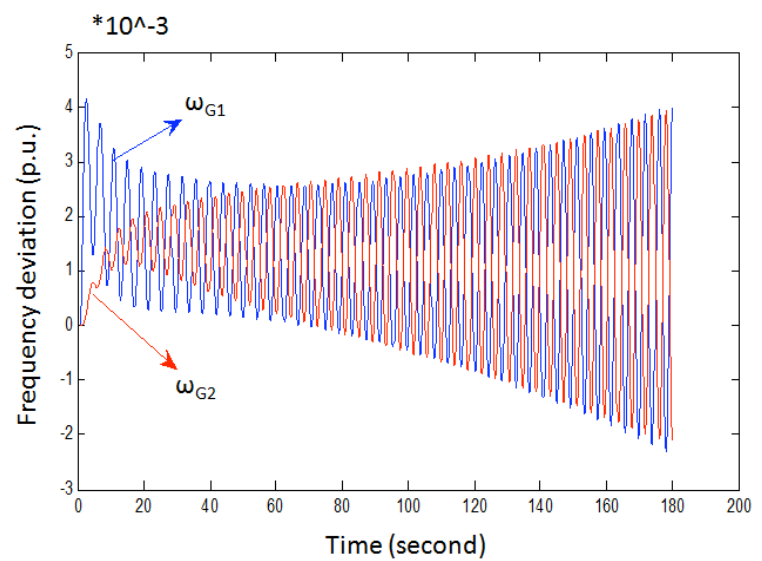

Fig. 3. Illustration of how the frequency deviation of the distribution system with two C-Ts placed at optimum locations diverge to infinite when small perturbation, equal to 0.1 p.u., occurs at bus 15 .

characteristics of decentralized control systems. This problem can be resolved only by optimally implementing centralized control systems.

\section{Centralized Control Design for Modern DISTRIBUTED ENERGY SYSTEMS}

Another method to warrant robustness of modern distribution systems with large penetration of MW-scale DG units is employing centralized control systems. In fact, centralized control systems potentially allow us to stabilize the 
whole system with minimum MW loss and with maximum efficiency. That is, the global optimum equilibrium can be extracted by this method.

In general, the necessary and sufficient condition for the small-signal stability of a system is satisfied when all the eigenvalues of the system matrix lie in the left-hand side of the complex plane [18]. In other words, all the eigenvalues of the system have negative real part or the system matrix is negative definite.

Furthermore, the sufficient condition for the stability of systems with centralized control design is fulfilled if the system matrix satisfies the Hicks Stability conditions. That is, all odd-order principle minors of the system matrix are negative and all even-order principal minors of the system matrix are positive [18]. Equation (9) demonstrates the Hicks Stability condition.

$$
(-1)^{i} \times\left|\begin{array}{cccc}
A_{11} & A_{12} & \cdots & A_{1 i} \\
A_{21} & A_{22} & \cdots & A_{2 i} \\
\vdots & \vdots & & \\
A_{i 1} & A_{i 2} & \cdots & A_{i i}
\end{array}\right|>0 \quad \forall i \in[1, n]
$$

In general, in order to warrant small-signal stability of electric power systems with centralized control systems, the whole system needs to be fully controllable and observable. That is, the controllability and observability matrices should be full rank. To this end, implementing high level of reliable centralized communication and observation is essential. This allows us to stabilize the system at the global optimal equilibrium point. The controllability and observabililty matrices are also calculated from the following equations [18]:

$$
\begin{gathered}
\mathrm{K}=\left[B A B \cdots A^{n-1} B\right] \\
\Omega=\left[\begin{array}{c}
C \\
C A \\
\vdots \\
C A^{n-1}
\end{array}\right]
\end{gathered}
$$

where $\mathrm{K}$ is the controllability matrix, $\Omega$ is defined as the observability matrix, $A$ is the system matrix, $B$ is the control matrix, and $C$ is the output matrix.

Figure 4 illustrates how dynamic behavior of the representative distribution system shown in Figure 1 has been improved by implementing an appropriate centralized control system. Eigenvalues of the system, before and after implementing the centralized control system have been presented in Appendix A. Equation (10) also represents the general mathematical formula of centralized control systems.

$$
\frac{d}{d t}\left[\begin{array}{c}
\bar{X}_{1} \\
\vdots
\end{array}\right]=\left[\begin{array}{ccc}
A_{11} & A_{12} & \cdots \\
A_{21} & A_{22} & \cdots \\
\vdots & \vdots & \ddots
\end{array}\right]\left[\begin{array}{c}
\bar{X}_{1} \\
\vdots
\end{array}\right]+\left[\begin{array}{c}
B_{1} \\
\vdots
\end{array}\right] \bar{U}
$$

where $\bar{U}$ is the vector of centralized control signals, calculated based on the desired poles of the full system. $K$ is also the gain matrix of the centralized control.

$$
\bar{U}=-K\left[\begin{array}{c}
\bar{X}_{1} \\
\vdots
\end{array}\right]
$$

In fact, centralized control systems can potentially stabilize the system at the globally optimum equilibrium point; however, these systems have some inherent drawbacks for future distribution systems. For instance, costs of stabilizing

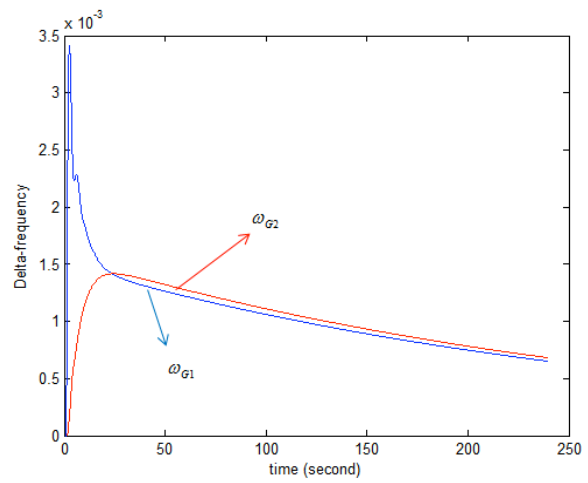

Fig. 4. Illustration of converging frequency deviations after implementing centralized control.

systems with centralized controls are often much higher than that of decentralized control systems. In addition, centralized controls are very complex since only one decision maker designs the whole system. Moreover, employing centralized control systems raise the issues of cyber security. Centralized control systems have also long communication times and therefore are less effective for small-signal stability purpose.

In general it is feasible to stabilize modern distribution systems both with decentralized or centralized control systems (locally or globally). However, the question automatically raises of which control strategy is more appropriate. A possible answer to this question is that choosing between these two strategies depends highly on existing system design, regulation and policy. If the system already has a hierarchical structure and the policy of the utility is to conserve this structure, then a centralized control system is likely to be a better option. On the other hand, if the system has been converted to a liberalized system and policy is in favor of liberalization, then a proper control strategy for the system would more likely be decentralized control.

\section{POLICY IMPliCATIONS AND CONCLUSIONS}

This paper has demonstrated that advanced centralized or decentralized frequency control systems are essential to increase robustness and stability of the future distribution systems; otherwise, a simple laissez-faire approach, under which private operators can simply install and operate DG units without any constraint, is likely to lead to significant stability problems. 
The technical findings demonstrate where larger DGs are located can have a significant role in stability of modern distribution systems. The analyses developed in this research also point out that strong electrical interaction between DGs, low damping magnitude of DGs, and low inertia of DGs are the major causes of small-signal frequency instabilities in distribution systems with large penetration of DGs. If the strength of electrical interaction between DG units is more than the damping magnitude of the slowest eigenmode of DGs, strong coupling between generators might lead to overall instability in the local distribution systems. The results also show that critical electrical distance between DGs is a function of DGs' and networks' parameters such as the network location of DGs, inertia of DGs, gain of G-Cs, number of DGs in the system and networks' topology and characteristics.

If new, more distributed generation systems are to become viable, either some level of centralized and coordinated advanced dynamic modeling and analysis or some form of smart and advanced decentralized control systems will be essential to warrant frequency stability of modern distribution systems. In addition, the technical tools must include advanced dynamic analysis tools in parallel with static optimization software to ensure both efficiency and robustness. However, choosing between these two controlstrategies raises questions about "who is in charge" and of tradeoffs between high communication and observation or advanced local control systems. The best solution will depend essentially on technical design and regulation policies of the system.

From this work it is concluded that first it is not always feasible to allow independent entitles to site MW-scale DG facilities wherever they choose if distribution systems are designed to sell significant amounts of power to the electric system and DGs are not equipped with advanced decentralized or centralized control systems. Second, frequency stability of modern distribution systems is very system-dependent

\section{APPENDIX A}

In this section eigen values of systems mentioned in sections III and IV are presented.

\section{TABLE A.1}

EIGENVALUES OF 30 BUS SYSTEM WHEN DGS ARE PLACED IN DIFFERENT LOCATIONS

\begin{tabular}{|c|c|}
\hline $\begin{array}{c}\text { DGs at buses 13 } \\
\text { and 14 (Optimum } \\
\text { Locations) }\end{array}$ & $\begin{array}{c}\text { DGs at buses 10 } \\
\text { and 14 (Stable } \\
\text { Case) }\end{array}$ \\
\hline-19.9943 & -19.9943 \\
-19.9943 & -19.9943 \\
$0.0081+1.5217 \mathrm{i}$ & $-0.0130+1.5029 \mathrm{i}$ \\
$0.0081-1.5217 \mathrm{i}$ & $-0.0130-1.5029 \mathrm{i}$ \\
$-0.0186+1.4984 \mathrm{i}$ & $-0.0184+1.4985 \mathrm{i}$ \\
$-0.0186-1.4984 \mathrm{i}$ & $-0.0184-1.4985 \mathrm{i}$ \\
-1.1387 & -1.1378 \\
-0.9989 & -1.1130 \\
-0.1969 & -0.0050 \\
-0.0037 & -0.0407 \\
\hline
\end{tabular}

TABLE A.2

EIGENVALUES OF AN UNSTABLE CASE, EIGENVALUES OF ITS SUB-SYSTEMS AND COUPLING VARIABLES

\begin{tabular}{|c|c|c|c|}
\hline $\begin{array}{l}\text { DGs at buses } 4 \\
\text { and } 5\end{array}$ & $\begin{array}{l}\text { Sub-system } 1 \\
\text { (DG at bus 4) }\end{array}$ & $\begin{array}{l}\text { Sub-system } 1 \\
\text { (DG at bus 5) }\end{array}$ & $\begin{array}{c}\text { Coupling } \\
\text { variable } \mathrm{Kp}_{12}\end{array}$ \\
\hline \multirow{3}{*}{$\begin{array}{c}-19.9943 \\
-19.9943 \\
0.0695+1.5217 \mathrm{i} \\
0.0695-1.5217 \mathrm{i} \\
-0.0161+1.4984 \mathrm{i} \\
-0.0161-1.4984 \mathrm{i} \\
-1.1274 \\
-0.6593+0.6914 \mathrm{i} \\
-0.6593-0.6914 \mathrm{i} \\
-0.0200\end{array}$} & \multirow{3}{*}{$\begin{array}{c}-19.9943 \\
0.0334+1.3170 \mathrm{i} \\
0.0334-1.3170 \mathrm{i} \\
-0.7300 \\
-0.5164\end{array}$} & \multirow{3}{*}{$\begin{array}{c}-19.9943 \\
0.0273+1.1450 \mathrm{i} \\
0.0273-1.1450 \mathrm{i} \\
-0.8347 \\
-0.3396\end{array}$} & -4.1399 \\
\hline & & & $\begin{array}{c}\text { Coupling } \\
\text { variable } \mathrm{Kp}_{21}\end{array}$ \\
\hline & & & -4.1053 \\
\hline
\end{tabular}

TABLE A.3

EIGENVALUES OF 30 BUS SYSTEM WHEN DGS EQUIPPED W/O CENTRALIZED CONTROL SYSTEMS

\begin{tabular}{|c|c|}
\hline Before Centralized Control & After Centralized Control \\
\hline-19.9943 & -19.9942 \\
-19.9943 & -19.9942 \\
$0.0081+1.5218 \mathrm{i}$ & $-0.5571+1.5245 \mathrm{i}$ \\
$0.0081-1.5218 \mathrm{i}$ & $-0.5571-1.5245 \mathrm{i}$ \\
$-0.0186+1.4984 \mathrm{i}$ & $-0.5738+1.5049 \mathrm{i}$ \\
$-0.0186-1.4984 \mathrm{i}$ & $-0.5738-1.5049 \mathrm{i}$ \\
-1.1386 & -1.2955 \\
-0.9987 & -1.1845 \\
-0.1973 & -0.1733 \\
-0.0039 & -0.0035 \\
\hline
\end{tabular}

\section{APPENDIX B}

Here the state-space dynamic model of combustion-turbine mentioned in section III is presented.

$\frac{d}{d t}\left[\begin{array}{l}\omega_{G} \\ V_{C E} \\ W_{F} \\ W_{F} d\end{array}\right]=\left[\begin{array}{cccc}\frac{-D}{M} & 0 & \frac{c}{M} & 0 \\ \frac{-K_{D}}{b} & \frac{-1}{b} & 0 & 0 \\ 0 & 0 & 0 & 1 \\ 0 & 1 & \frac{-\delta}{\alpha} & \frac{-\beta}{\alpha}\end{array}\right]\left[\begin{array}{l}\omega_{G} \\ V_{C E} \\ W_{F} \\ W_{F} d\end{array}\right]+\left[\begin{array}{c}\frac{-1}{M} \\ 0 \\ 0 \\ 0\end{array}\right] P_{G}+\left[\begin{array}{c}0 \\ \frac{K_{D}}{b} \\ 0 \\ 0\end{array}\right] \omega^{r e f}$

In this model, $\omega_{\mathrm{G}}$ is frequency, $V_{C E}$ is fuel control, $W_{F}$ is fuel flow and $W_{F} d$ is the derivative of fuel flow. $M$ and $D$ are the inertia and damping coefficients respectively. $a, b$ and $c$ are transfer function coefficients for the fuel system, and $K_{D}$ is the governor gain. $\beta$ and $\delta$ are algebraic functions of the parameters, defined as $\beta=b+c \tau_{F}$ and $\delta=c+a K_{F}$, where $\tau_{F}$ is the fuel system time constant, and $K_{F}$ is the fuel system feedback gain. Numerical values of combustion turbine parameters are presented in Table B.1. These equations are derived and

TABLE B. 1

PARAMETERS OF COMBUSTION-TURBINE GENERATOR [4]. COEFFICIENTS ARE IN PER UNIT

\begin{tabular}{|ll|ll|}
\hline \multicolumn{4}{|c|}{ Combustion-Turbine parameters } \\
\hline $\mathrm{M}$ & 11.5 & $\mathrm{\alpha}$ & 0.45 \\
$\mathrm{D}$ & 2.0 & $\mathrm{a}$ & 1.0 \\
$\mathrm{c}$ & 1.0 & $\tau_{\mathrm{F}}$ & 0.40 \\
$\mathrm{~K}_{\mathrm{D}}$ & 25.0 & $\mathrm{~K}_{\mathrm{F}}$ & 0.0 \\
$\mathrm{~b}$ & 0.05 & & \\
\hline
\end{tabular}


simplified from the equations and models found in [19], [20] and [21] and used for the first time in [4].

\section{ACKNOWLEDGMENT}

The authors greatly appreciates the help by the M.I.T. Professor Jeffrey Lang in running the AC OPF program owned by the New Electricity Transmission Software Solutions (NETSS), Inc. which was needed to compute the optimal voltage profile for all possible combinations of candidate DGs. Financial support for this work was provided by the Portugal-Carnegie Mellon joint program.

\section{REFERENCES}

[1] H. Zerriffi, H. Dowlatabadi, J. Apt "Electric Power Systems under Stress: An Evaluation of Centralized versus Distributed System Architectures", Doctor of Philosophy, Engineering and Public Policy, Carnegie Mellon University, 2004.

[2] N. Straub, and P. Behr, "Energy Regulatory Chief Says New Coal, Nuclear Plants May Be Unnecessary," Energy \& Environment, April 22, 2009.

[3] J. Cardell and M. Ilić, "Maintaining Stability with Distributed Generation in the Restructured Electric Power Industry", Proceedings of the IEEE PES GM, Boulder, CO, June 2004.

[4] J. Cardell and M. Ilić, and Tabors, R. D. "Integrating Small Scale Distributed Generation into a Deregulated Market: Control Strategies and Price Feedback", Laboratory for Electromagnetic and Electronic Systems, Massachusetts Institute of Technology, 1998.

[5] M. Ângelo and P. Lopes, "Simultaneous Tuning of Power System Stabilizers Installed in DFIG-Based Wind Generation", Power Tech Proceedings, Lausanne, Switzerland, July 2007.

[6] R. T. Guttromson, "Modeling Distributed Energy Resource Dynamics on the Transmission System," IEEE Trans. on Power Systems, Vol. 17, No. 4, Nov. 2002.

[7] M. K. Donnelly, J. E. Dagle, D. J. Trudnowski, and G. J. Rogers, "Impacts of the Distributed Utility on Transmission System stability," IEEE Trans. on Power Systems, Vol. 11, No. 2, May 1996.

[8] A. L'Abbate, S. Peteves, and G. Fulli, "Challenges for DG integration in Europe - status and potentials vary by country," Cogeneration \& On-Site Power Production Magazine, 10(4), July 2009.

[9] A. H. El-Abiad, "Power Systems Analysis and Planning", Hemisphere Pub. Corp., 1983.

[10] W. Katzenstein and J. Apt, "Incorporating Wind into a Natural-gas Turbine Baseload Power System Increases $\mathrm{NOx}$ and $\mathrm{CO}_{2}$ Emissions from the Gas Turbines", Future Energy Systems Conf., Carnegie Mellon University, March 2008.

[11] J. Apt, L. Lave, and S. Pattanariyankool, "A National Renewable Portfolio Standard? Not Practical" Issues in Science and Technology", Vol. XXV, No. 1, Fall 2008.

[12] W. Rickerson, F. Bennhold, and R. J. Bradbury, "Feed-in Tariffs and Renewable Energy the USA - a Policy Up-Date", Report by the World Future Council, Heinrich Boll, May 2008.

[13] M. Ilić and J. Zaborszky, "Dynamics and Control of Large Electric Power Systems", John Wiley \& Sons, 2000.

[14] G. Rogers, "Power System Oscillations", Boston, Kluwer Academic Publishers, 2000

[15] M. A. Pai, D. P. S. Gupta, and K. R. Padiyar, "Small Signal Analysis of Power Systems", Harrow, Alpha Science International, 2004.

[16] X. Liu and M. Ilic "Structural Modeling and Hierarchical Control of Large-Scale Electric Power Systems," Doctor of Philosophy, Massachusetts Institute of Technology, April 1994.

[17] D. G. Feingold and R. S. Varga, "Block Diagonally Dominant Matrices and Generalizations of the Gerschgorin Circle Theorem," Pacific J. Math. 12(4) pp. 1241-1250, 1962.

[18] D. Siljak, Large-Scale Dynamic Systems: Stability and Structure, Dover Publications, Nov. 2007.

[19] L. N. Hannett and Afzal Khan, "Combustion Turbine Dynamic Model Validation form tests", IEEE Transactions on Power Systems, 8(1), February 1983.
[20] Louis N. Hannett, George Jee, and B. Fardanesh, "A Governor/Turbine Model for a Twin-Shaft Combustion Turbine", IEEE Transactions on Power Systems, 10(1), February 1995.

[21] W. I. Rowen, "Simplified Mathematical Representations of Heavy-Duty Gas Turbines", Journal of Engineering for Power, 105, Oct 1983.

[22] M. Calović, "Dynamic State-Space Models of Electric Power Systems", Technical report, University of Illinois, Urbana, Illinois, 1971.

[23] Radial Distribution test Feeders. IEEE Transactions on Power Systems, 6(3), August 1991. IEEE Distribution Planning Working Group Report.

[24] http://grouper.ieee.org/groups/scc21/1547/1547 index.html

[25] M. Honarvar Nazari and M. Ilić, "Technical Challenges in Modernizing Distribution Electric Power Systems with Large Number of Distributed Generators", Proceedings of the IEEE PES PowerTech, Bucharest, Romania, June 2009.

\section{BIOGRAPHIES}

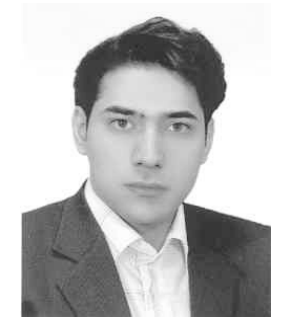

Masoud Honarvar Nazari received his B.Sc. degree in Electrical Engineering from KNTU (Khaje Nasir Al-Deen Toosi) University of Technology in 2003, and the M.Sc. degree in Energy Systems Engineering from Sharif University of Technology (SUT), Iran, in 2005. He was admitted as a member of talented students club in Iran at 2005-6. He is now a joint Ph.D. student of Engineering and Public Policy and Electrical and Computer Engineering at Carnegie Mellon University (CMU), U.S. He is also pursuing a dual $\mathrm{PhD}$ in Electrical Engineering Department of Porto University. In addition, he has awarded the international FCT fellowship in 2007. He has many papers in the subject of Distributed Generation. His research interests include Dynamic modeling and design of control algorithms for Future Electric Energy Systems; Policy implication and regulation design of modernizing electric power systems to ensure efficiency and robustness, and; re-framing policy design and technical standards of Distributed Generation to avoid technical problems.

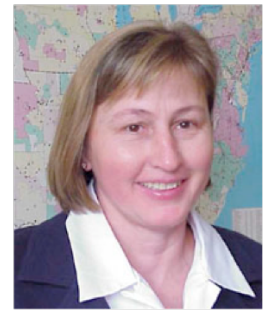

Marija Ilic is a Professor at Carnegie Mellon University, Pittsburgh, PA, with a joint appointment in the Electrical and Computer Engineering and Engineering and Public Policy Departments. She is also the Honorary Chaired Professor for Control of Future Electricity Network Operations in the Faculty of Technology, Policy and Management at Delft University of Technology, Delft, The Netherlands. She was an assistant professor at Cornell University, Ithaca, NY, and tenured Associate Professor at the University of Illinois at UrbanaChampaign. She was then a Senior Research Scientist in Department of Electrical Engineering and Computer Science, Massachusetts Institute of Technology, Cambridge, from 1987 to 2002. She has 30 years of experience in teaching and research in the area of electrical power system modeling and control. Her main interest is in the systems aspects of operations, planning, and economics of the electric power industry. She has co-authored several books in her field of interest. Prof. Ilic is an IEEE Fellow.

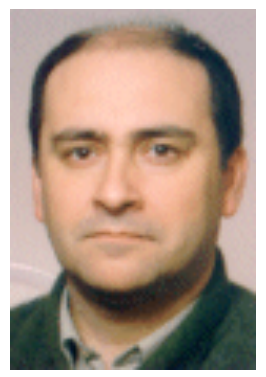

J. A. Peças Lopes (M'80-SM'94) received the electrical engineering degree and the Ph.D. degree in electrical engineering from the University of Porto, Porto, Portugal, in 1981 and 1988, respectively. In 1996, he received the Aggregation degree from the University of Porto. Currently, he is Full Professor in the Department of Electrical Engineering of the Faculty of Engineering of University of Porto, where he is Director of the Sustainable Energy Systems $\mathrm{PhD}$ course. He is also one of the Directors of the Instituto de Engenharia de Sistemas e Computadores do Porto (INESC Porto). 